\title{
Increased Serum Pentraxin 3 Levels are Associated with Poor Prognosis of Hepatitis B Virus-Related Hepatocellular Carcinoma
}

\author{
Qunying Han' \\ Huan Deng' \\ Xiude Fan (1D) \\ Xiaoyun Wang' \\ Xiaoge Zhang' \\ Kun Zhang' \\ $\mathrm{Na} \mathrm{Li}{ }^{\prime}$ \\ Yi Lv ${ }^{2,3}$ \\ Zhengwen Liu (iD) ${ }^{1,3}$
}

'Department of Infectious Diseases, First Affiliated Hospital of Xi'an Jiaotong University, Xi'an, Shaanxi, People's Republic of China; ${ }^{2}$ Department of Hepatobiliary Surgery, First Affiliated Hospital of Xi'an Jiaotong University, Xi'an, Shaanxi, People's Republic of China; ${ }^{3}$ Institute of Advanced Surgical Technology and Engineering, Xi'an Jiaotong University, Xi'an, Shaanxi, People's Republic of China
Background: Hepatocellular carcinoma (HCC) is a major cause of liver-related mortality. Serum pentraxin 3 (PTX3) has been revealed to be associated with the development of hepatitis B virus (HBV)-related HCC. This study evaluated whether serum PTX3 is related to the survival of HBV-related HCC patients.

Methods: One hundred and seven patients with HBV-related HCC were included. Baseline serum PTX3 levels were quantified using quantitative immunoassay. The HCC patients were followed-up for a median of 24 months and divided into high serum PTX3 level and low PTX3 level groups according to the baseline serum PTX3 levels. The overall survivals of the HBV-related HCC patients according to the serum PTX3 levels were compared. Factors potentially influencing the prognosis of the patients with HBV-related HCC were analyzed. Results: HCC patients with high serum PTX3 levels [PTX3 $>9.25 \mathrm{ng} / \mathrm{mL}(\mathrm{n}=85)]$ had a shorter overall survival time than HCC patients with low serum PTX3 levels [PTX3 $\leq 9.25 \mathrm{ng} / \mathrm{mL}(\mathrm{n}=22)]$ $(P=0.049)$. HCC patients with serum PTX3 levels between $>9.25 \mathrm{ng} / \mathrm{mL}$ and $\leq 9.25 \mathrm{ng} / \mathrm{mL}$ had significant difference in HCC histology grade. Multivariate analysis showed that PTX3 level was an independent risk factor related to the overall survival of HCC patients (hazard ratio: $1.058,95 \%$ confidence interval: $1.031-1.085, P<0.001)$.

Conclusion: These results support the involvement of PTX3 in the disease progression of HCC and suggest the potential of using serum PTX3 levels as a biomarker for the prognostic prediction of HBV-related HCC patients.

Keywords: hepatocellular carcinoma, pentraxin 3, chronic HBV infection, survival, prognosis

\section{Introduction}

Hepatocellular carcinoma (HCC) is a leading cause of cancer-related deaths worldwide. ${ }^{1}$ Hepatitis B virus (HBV) is one of the major causative agents associated with $\mathrm{HCC}$ development worldwide ${ }^{2-4}$ and the most common causative agent of $\mathrm{HCC}$ in $\mathrm{China}^{5}$. The lack of biomarkers for the early detection and approaches for the cure significantly affects the prognosis of HCC. ${ }^{6,7}$ Among the various serum markers, alpha-fetoprotein (AFP) has been the mostly used biomarker for HCC detection and prognosis. ${ }^{8}$ However, the sensitivity and specificity of AFP for the detection and prognosis of HCC, especially for AFP negative HCC, remain unsatisfactory. ${ }^{8-10}$ Therefore, to improve the management of HCC patients, the identification of new biomarkers remains an urgent need.

Pentraxin 3 (PTX3), also known as tumor necrosis factor-stimulated gene 14 (TSG14), is a long pentraxin of the pentraxin superfamily. ${ }^{11,12}$ It is a typical acute-phase protein
Correspondence: Zhengwen Liu Department of Infectious Diseases, First Affiliated Hospital of Xi'an Jiaotong University, No. 277 Yanta West Road, Xi'an, 7I006I, Shaanxi Province, People's Republic of China

Email liuzhengwenII3@xjtu.edu.cn 
produced by a variety of cells, such as leukocytes, dendritic cells, and endothelial cells, in response to local inflammation. ${ }^{13-15}$ PTX3 plays a critical homeostatic role in linking innate immunity, tissue repair, inflammation, and cancer. $^{16,17}$ Furthermore, PTX3 is involved in the regulation of the biological behaviors such as proliferation, angiogenesis and metastasis associated with malignancies. ${ }^{17}$ In terms of HCC, studies have showed that PTX3 could enhance HCC progression. ${ }^{18}$ Elevated PTX3 expression in tumor tissues is associated with poor prognosis in HCC patients. ${ }^{18}$ Serum PTX3 is a risk factor for HCC development in chronic hepatitis $\mathrm{C}$ virus (HCV) infection. ${ }^{19}$ In $\mathrm{HBV}$ infection, serum PTX3 level is associated with the development of HCC and has a diagnostic value for HCC including AFP negative HCC. ${ }^{20}$

However, whether serum PTX3 levels are related to the prognosis of HBV-related HCC patients remains unknown. This study, therefore, investigated the association of serum PTX3 levels with the overall survival of patients with HBV-related HCC.

\section{Methods}

\section{Patients}

Patients with HBV-related HCC were recruited from the First Affiliated Hospital of Xi' an Jiaotong University. HBV-related $\mathrm{HCC}$ was diagnosed based on the evidence of chronic HBV infection and the characteristics of ultrasound, computed tomography and/or magnetic resonance and/or histopathology. ${ }^{21}$ HCC stage was defined based the Barcelona Clinic Liver Cancer (BCLC) staging system. ${ }^{22}$ Patients aged less than 18 years were excluded. Patients with liver diseases related to other etiologies including nonalcoholic fatty liver disease, alcoholic liver disease, and drug-induced liver injury were excluded. Patients with autoimmune diseases such as autoimmune hepatitis, primary biliary cirrhosis, primary sclerosing cholangitis and systemic lupus erythematosus, and severe disorders of other systems were also excluded. The patients were followed-up at 1- to 3-month intervals according to the disease condition.

This study conformed with the Declaration of Helsinki. The study approval was obtained from the Ethics Committee of the First Affiliated Hospital of Xi'an Jiaotong University (2013-086). Each patient gave informed consent.

\section{Determination of Laboratory Parameters and Serum PTX3 Levels}

Blood samples at baseline were obtained from each patient. Blood tests and biochemistry including biochemical liver functions were determined at the clinical laboratory of the hospital. Serum HBV DNA was determined using quantitative real-time polymerase chain reaction. HBsAg, anti-HBs, $\mathrm{HBeAg}$, anti-HBe, and anti-HBc were assayed by enzymelinked immunosorbent assay. Serum AFP levels (ng/mL) were examined by automated Eleceyes (Hoffman-La Roche Ltd., Basel, Switzerland). Serum PTX3 levels were quantified using The Quantikine Human Pentraxin 3/TSG-14 Immunoassay (R\&D Systems China Co., Ltd. Shanghai, China) as described previously. $^{20}$

\section{Statistical Analysis}

Statistical analysis was conducted using SPSS 24.0 software. Quantitative variables were expressed as mean and standard deviation (SD) or median (range). Categorical variables were expressed as absolute or relative frequencies. Continuous variables were compared using the analysis of variance. Categorical variables were compared by ChiSquare test. The prognosis of HBV-related HCC patients according to the serum PTX3 levels was compared by Kaplan-Meier curve and tested by Log rank test. The analysis of factors potentially influencing the prognosis of patients with HBV-related HCC was carried out by univariate and multivariate Cox regression analysis. $P$ values less than 0.05 were regarded as statistically significant.

\section{Results}

\section{Characteristics of the Participants}

One hundred and seven patients with HBV-related HCC were recruited. The demographic and laboratory findings in the 107 patients were shown in Table 1. Of the 107 patients, 92 were males and 15 were females. The age of the patients ranged from 18 to 77 years (average $49.4 \pm 10.90$ years). The patients were followed-up for a median of 24 months (1-73 months, mean 28.44 months, Table 1).

\section{Serum PTX3 Levels and Overall Survival of HCC Patients}

According to the cut-off value of serum PTX3 for HBVrelated $\mathrm{HCC}$ at baseline determined in previous study, ${ }^{20}$ the patients were divided into high serum PTX3 level [PTX3 > $9.25 \mathrm{ng} / \mathrm{mL}(\mathrm{n}=85)]$ and low serum PTX3 level [PTX3 $\leq$ $9.25 \mathrm{ng} / \mathrm{mL}(\mathrm{n}=22)]$ groups. The baseline characteristics between patients with PTX3 $\leq 9.25 \mathrm{ng} / \mathrm{mL}$ and those with PTX3 $>9.25 \mathrm{ng} / \mathrm{mL}$ are shown in Table 2. The gender, age, HBV DNA, ALT, AST, total bilirubin, albumin, AFP and tumor diameter between the two groups of patients showed 
Table I Characteristics of the 107 HCC Patients

\begin{tabular}{|l|l|}
\hline Variables & \\
\hline Gender (male/female) & $92 / 15$ \\
Age (years) & $49.4 \pm 10.90(18-77)$ \\
HBV DNA (IU/mL, log) & $4.90 \pm 1.50$ \\
ALT (IU/L) & $54(33-102)$ \\
AST (IU/L) & $70(35-150)$ \\
TBIL ( $\mu \mathrm{mol} / \mathrm{L})$ & $26.2(15.2-46.7)$ \\
Albumin $(g / L)$ & $33(29.7-39.1)$ \\
AFP $(\mathrm{ng} / \mathrm{mL})$ & $97.4(16.8-4886)$ \\
PTX3 $(\mathrm{ng} / \mathrm{mL})$ & $15.6(9.6-24.3)$ \\
\hline
\end{tabular}

Abbreviations: HBV, hepatitis B virus; HCC, hepatocellular carcinoma; ALT, alanine aminotransferase; AST, aspartate aminotransferase; TBIL, total bilirubin; AFP, $\alpha$-fetoprotein; PTX3, pentraxin 3.

no significant differences. The metastasis occurrence and BCLC stage between the two groups also showed no significant differences. However, the histological differentiation between the low serum PTX3 and high serum PTX3 groups was significantly different $(P<0.001$, Table 2$)$. Patients with high serum PTX3 levels tended to have more low grade differentiation HCC compared with patients with low serum PTX3 levels.

The overall survivals of the patients according to PTX3 levels were shown in Figure 1. HCC patients with high serum PTX3 levels (PTX3 $>9.25 \mathrm{ng} / \mathrm{mL}$ ) had a significantly shorter overall survival time than HCC patients with low serum PTX3 levels $(\mathrm{PTX} 3 \leq 9.25 \mathrm{ng} / \mathrm{mL})(P=0.049$, Figure 1$)$.

\section{Factors Associated with the Overall Survival of HCC Patients}

Univariate analysis showed that higher total bilirubin, lower albumin, and higher PTX3 levels were significant

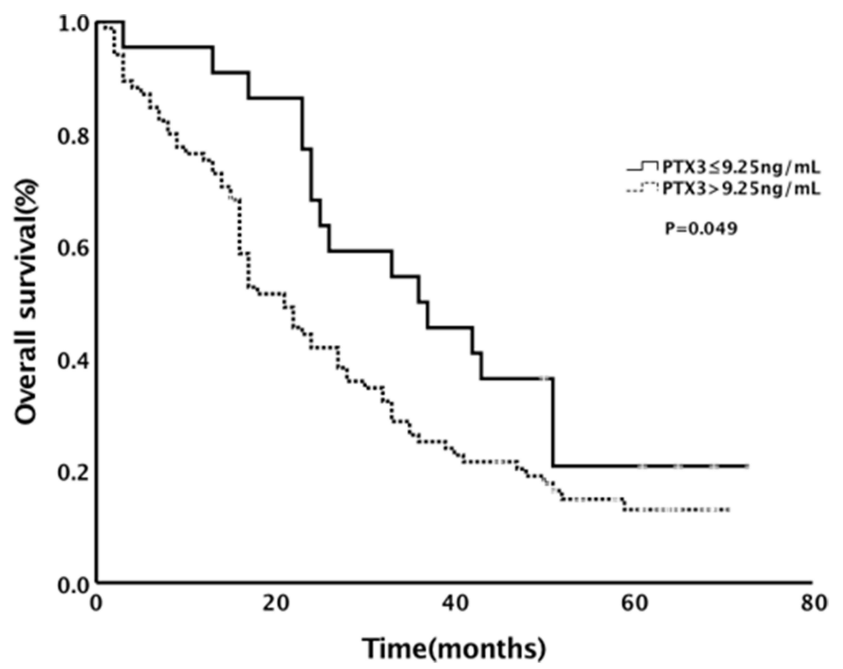

Figure I Overall survivals of hepatocellular carcinoma patients according to serum pentraxin 3 (PTX3) levels.

risk factors associated with shorter overall survival of HCC patients $(P<0.001, P=0.041$ and $P<0.001$, respectively). Multivariate analysis showed that total bilirubin [hazard ratio (HR): 1.933, 95\% confidence interval (CI): 1.075-3.536] and serum PTX3 (HR: 1.058, 95\% CI: 1.031-1.085) levels were independent risk factors related to the overall survival of HCC patients $(P=0.032$ and $P<$ 0.001 , respectively, Table 3 , Figure 2).

\section{Discussion}

HCC remains one of the most deadly malignancies, and the prognosis of $\mathrm{HCC}$ is poor in many patients. ${ }^{5}$ Clinically, prognostic biomarkers are useful for predicting the progression of the disease and may be used to plan the

Table 2 Comparison of the Baseline Characteristics Between Patients with PTX3 $59.25 \mathrm{ng} / \mathrm{Ml}$ and Patients with PTX3 >9.25ng/Ml

\begin{tabular}{|c|c|c|c|}
\hline Variables & PTX3 $\leq 9.25 \mathrm{ng} / \mathrm{mL}(\mathrm{n}=22)$ & PTX3 > 9.25ng/mL $(n=85)$ & $\boldsymbol{P}$ \\
\hline Gender (male/female) & $20 / 2$ & $72 / 13$ & 0.455 \\
\hline Age (years) & $52.8 \pm 11.26$ & $48.56 \pm 10.69$ & 0.080 \\
\hline HBV DNA (IU/mL, log) & $4.7 I \pm I .60$ & $4.95 \pm 1.48$ & 0.481 \\
\hline ALT (IU/L) & $55(7-765)$ & $53(10-603)$ & 0.326 \\
\hline AST (IU/L) & $53(23-647)$ & $74(15-1348)$ & 0.179 \\
\hline TBIL $(\mu \mathrm{mol} / \mathrm{L})$ & $26.2(12.6-409.4)$ & $25.3(1.5-727.2)$ & 0.799 \\
\hline Albumin $(g / L)$ & 34.1 (23.5-49) & $33.3(2||-5 \mid .3)$. & 0.445 \\
\hline $\operatorname{AFP}(\mathrm{ng} / \mathrm{mL})$ & $99.4(2.51-84,150)$ & $97.4(2.01-325,900)$ & 0.954 \\
\hline Tumor diameter $(\mathrm{mm})$ & $35.5 \pm 13.1$ & $34.7 \pm 12.7$ & 0.981 \\
\hline Metastasis (yes/no) & $17 / 5$ & $65 / 20$ & 0.937 \\
\hline $\mathrm{BCLC}$ stage $(0 / \mathrm{A} / \mathrm{B} / \mathrm{C} / \mathrm{D})$ & $3 / 13 / 4 / 2 / 0$ & $14 / 38 / 13 / 11 / 9$ & 0.481 \\
\hline Histology (I/II/III) & $3 / 3 / 7$ & $14 / 16 / 0$ & $<0.001$ \\
\hline
\end{tabular}

Abbreviations: HBV, hepatitis B virus; HCC, hepatocellular carcinoma; ALT, alanine aminotransferase; AST, aspartate aminotransferase; TBIL, total bilirubin; AFP, $\alpha$ fetoprotein; PTX3, pentraxin 3. 
Table 3 Univariate and Multivariate Analysis of Factors Associated with the Overall Survival of HCC Patients

\begin{tabular}{|c|c|c|c|c|c|c|c|}
\hline & \multirow[t]{2}{*}{$\mathbf{n}$} & \multicolumn{3}{|c|}{ Overall Survival (\%) } & \multirow{2}{*}{$\begin{array}{l}\text { Univariate Analysis } \\
\mathbf{P}\end{array}$} & \multicolumn{2}{|c|}{ Multivariate Analysis } \\
\hline & & I Year & 2 Year & 3 Year & & HR (95\% Cl) & $P$ \\
\hline Gender & & & & & 0.289 & $\mathrm{I} .050(0.53 \mathrm{I}-2.074)$ & 0.889 \\
\hline Male & 92 & 83 & 32 & 15 & & & \\
\hline Female & 15 & 60 & 20 & 13 & & & \\
\hline Age (years) & & & & & 0.855 & $1.005(0.640-1.579)$ & 0.982 \\
\hline$\leq 55$ & 70 & 80 & 34 & 14 & & & \\
\hline$>55$ & 37 & 78 & 24 & 16 & & & \\
\hline HBV DNA (IU/mL) & & & & & 0.874 & $1.006(0.858-1.180)$ & 0.937 \\
\hline$\leq 10^{4}$ & 59 & 83 & 36 & 13 & & & \\
\hline$>10^{4}$ & 48 & 78 & 28 & 15 & & & \\
\hline ALT (IU/L) & & & & & 0.524 & I. $174(0.66 \mathrm{I}-2.084)$ & 0.584 \\
\hline$\leq 40$ & 36 & 89 & 37 & 18 & & & \\
\hline$>40$ & 71 & 75 & 27 & 13 & & & \\
\hline AST (IU/L) & & & & & 0.150 & $1.087(0.572-2.066)$ & 0.798 \\
\hline$\leq 40$ & 29 & 97 & 36 & 28 & & & \\
\hline$>40$ & 78 & 73 & 28 & 10 & & & \\
\hline TBIL $(\mu \mathrm{mol} / \mathrm{L})$ & & & & & $<0.001$ & $1.933(1.075-3.536)$ & 0.032 \\
\hline$\leq 40$ & 74 & 93 & 38 & 18 & & & \\
\hline$>40$ & 33 & 47 & 13 & 6 & & & \\
\hline Albumin $(g / L)$ & & & & & 0.041 & $0.68 \mid(0.399-1.163)$ & 0.159 \\
\hline$\leq 32$ & 48 & 75 & 27 & 7 & & & \\
\hline$>32$ & 59 & 83 & 33 & 21 & & & \\
\hline $\operatorname{AFP}(\mathrm{ng} / \mathrm{mL})$ & & & & & 0.104 & $1.348(0.85 \mathrm{I}-2.135)$ & 0.203 \\
\hline$\leq 200$ & 58 & 84 & 38 & 14 & & & \\
\hline$>200$ & 49 & 73 & 21 & 15 & & & \\
\hline PTX3 (ng/mL) & & & & & $<0.001$ & $1.058(1.031-1.085)$ & $<0.001$ \\
\hline$\leq 9.25$ & 22 & 95 & 50 & 21 & & & \\
\hline$>9.25$ & 85 & 75 & 25 & 13 & & & \\
\hline MELD score & & & & & 0.524 & I.097 (0.487-2.47I) & 0.823 \\
\hline$\leq 15$ & 93 & 84 & 32 & 13 & & & \\
\hline$>15$ & 14 & 50 & 21 & 21 & & & \\
\hline Child-pugh grade & & & & & 0.061 & I. $128(0.655-2.267)$ & 0.533 \\
\hline A & 55 & 95 & 35 & 18 & & & \\
\hline$B+C$ & 52 & 73 & 29 & 14 & & & \\
\hline
\end{tabular}

Abbreviations: HBV, hepatitis B virus; HCC, hepatocellular carcinoma; ALT, alanine aminotransferase; AST, aspartate aminotransferase; TBIL, total bilirubin; AFP, $\alpha$ fetoprotein; PTX3, pentraxin 3; HR, hazard ratio; $95 \% \mathrm{Cl}, 95 \%$ confidence interval.

management strategies for the patients. Therefore, the identification of prognostic biomarkers is of important clinical value for HCC.

Previous study showed that high PTX3 level was an independent risk factor of $\mathrm{HCC}$ and related to $\mathrm{HCC}$ differentiation, and serum PTX3 levels could highly discriminate $\mathrm{HCC}$ from non $\mathrm{HCC}$ diseases in chronic $\mathrm{HBV}$ infection. $^{20}$ This study evaluated the role of serum PTX3 levels in the prognosis of HBV-related HCC patients, showing that increased serum PTX3 levels were associated with poor prognosis of HCC patients. These results support the involvement of PTX3 in the development and progression of $\mathrm{HBV}$-related $\mathrm{HCC}$, suggesting that the determination of PTX3 levels may possess not only a diagnostic value but also a prognostic value for HBVrelated $\mathrm{HCC}$. 


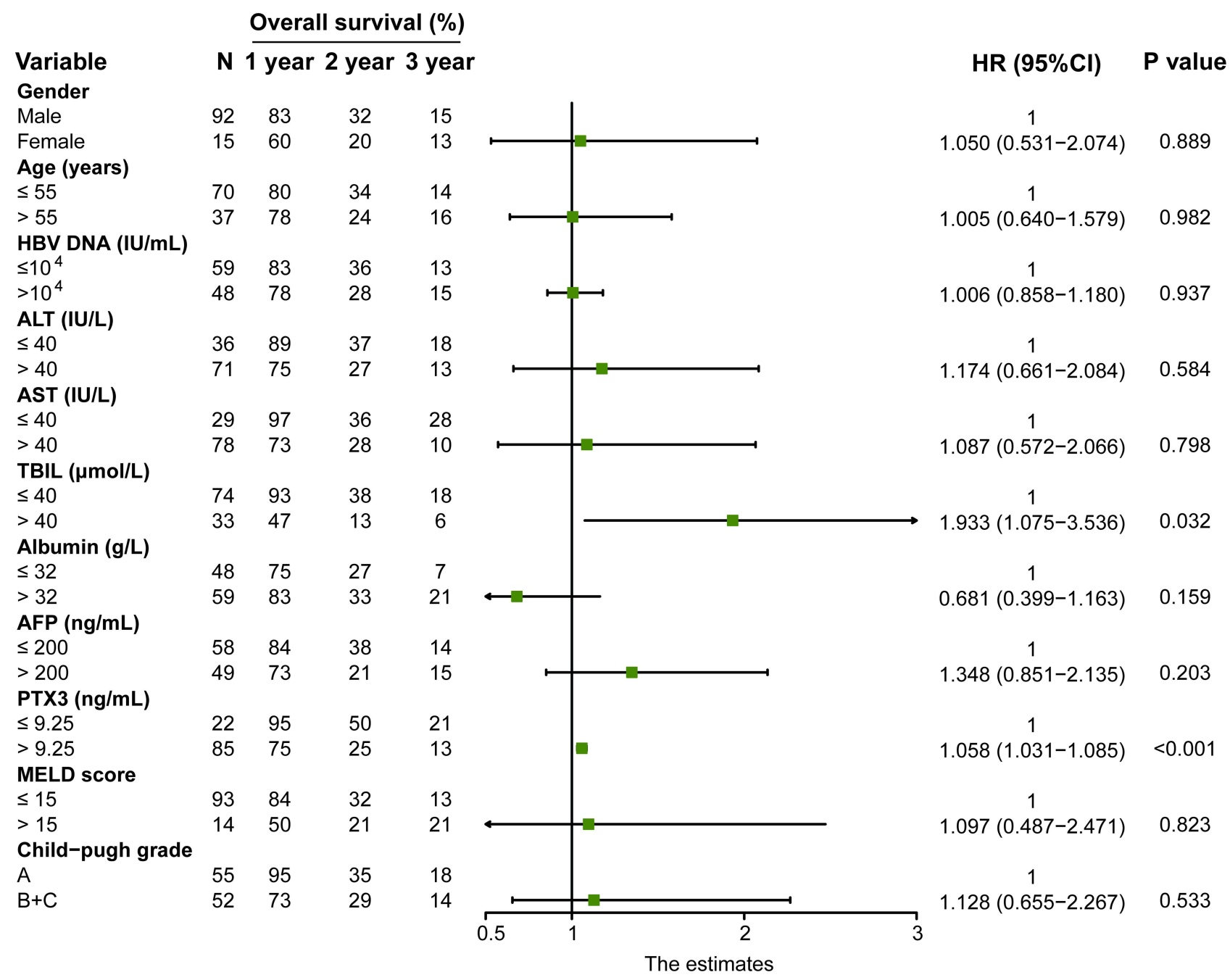

Figure 2 Associations of factors with the overall survival of HCC patients by multivariate Cox regression analysis.

Abbreviations: HBV, hepatitis B virus; HCC, hepatocellular carcinoma; ALT, alanine aminotransferase; AST, aspartate aminotransferase; TBIL, total bilirubin; AFP, $\alpha-$ fetoprotein; PTX3, pentraxin 3; HR, hazard ratio; $95 \% \mathrm{Cl}, 95 \%$ confidence interval.

The mechanisms of PTX3 in carcinogenesis and tumor progression have not been completely elucidated. Chronic inflammation is a key factor in the initiation and progression of cancer. ${ }^{23,24}$ PTX3 plays a complex regulatory role in inflammation including cancer-related inflammation. ${ }^{16}$ Innate immune responses including humoral innate immune responses are involved in cancer. ${ }^{24,25}$ PTX3, a evolutionarily conserved humoral pattern recognition molecule, ${ }^{13,26}$ links innate immunity, inflammation, tissue repair, and cancer ${ }^{16}$ and may impose important influences on tumor initiation, angiogenesis and metastasis via immune-regulation. $^{27}$ Increased PTX3 expression has been indicated to be related to poor prognosis in many cancers including breast, ${ }^{28}$ lung, ${ }^{29}$ gastric, ${ }^{30}$ pancreatic $^{31}$ and prostate ${ }^{32}$ cancers. In HCC, inflammation is an essential constituent of the tumor microenvironment that remarkably influences HCC development and progression. Immunologic mechanisms including humoral immunity play a critical role in the development and progression of HCC. ${ }^{33}$ PTX3 was also shown to be significantly related to liver disease severity and malignant biological behaviors including high-serum levels of aminotransferases, $\gamma$ glutamyl transferase and total bilirubin in HCV-related HCC patients. ${ }^{34}$ The strong correlation between PTX3 overexpression and HCC suggests the role of PTX3 in immunologic dysregulation and inflammation during the hepatocarcinogenesis and HCC progression. ${ }^{18-20,34}$ It should be noted that there is a study showing that PTX3 is not related to disease severity in cirrhosis and HCC patients. ${ }^{35}$ However, this study included relatively small number of cirrhosis $(n=35)$ and HCC $(n=31)$ patients. ${ }^{35}$ More other studies confirmed the potential of PTX3 as 
a biomarker in evaluating the disease severity and prognosis of cirrhosis $^{36}$ and HCC. ${ }^{18-20,34}$ The present study showed that increased serum PTX3 levels were associated with poor prognosis of HBV-related HCC and high PTX3 level was an independent factor associated with a reduced survival time of HCC patients. These findings added further information for the role of serum PTX3 in predicting the prognosis of malignancies.

AFP is the most commonly used biomarker of HCC but it is not suitable to detect and predict the prognosis of AFP-negative HCC. ${ }^{37}$ The present study showed that serum PTX3 levels are associated with the prognosis of HCC, suggesting the potential of applying serum PTX3 levels as a prognostic biomarker for $\mathrm{HBV}$-related $\mathrm{HCC}$ including AFP-negative HCC.

This study has several limitations. The sample size of the study is small. The study was carried out with no validation group and subgroup analysis according to therapeutic approaches. The HCC in the analyzed patients was only associated with HBV. Therefore, further studies are needed to validate the findings of this study.

In conclusion, this study showed that elevated serum PTX3 levels are associated with poor prognosis of HBVrelated $\mathrm{HCC}$, supporting the involvement of PTX3 in the disease progression and the potential of using serum PTX3 as a prognostic biomarker for HCC including AFP-negative HCC. Studies in large sample size of HCC patients related to various etiologies are required to confirm these results.

\section{Acknowledgments}

We thank all the participants of the study for their cooperation during the study.

\section{Funding}

This study was supported in part by grant from the National Natural Science Foundation of China (Grant no. 81371798). The funder plays no role in the study design, data collection and analysis, the manuscript preparation and decision to publish.

\section{Disclosure}

The authors report no conflicts of interest in this work.

\section{References}

1. Bray F, Ferlay J, Soerjomataram I, Siegel RL, Torre LA, Jemal A. Global cancer statistics 2018: GLOBOCAN estimates of incidence and mortality worldwide for 36 cancers in 185 countries. CA Cancer J Clin. 2018;68(6):394-424. doi:10.3322/caac.21492
2. Schweitzer A, Horn J, Mikolajczyk RT, Krause G, Ott JJ. Estimations of worldwide prevalence of chronic hepatitis B virus infection: a systematic review of data published between 1965 and 2013. Lancet. 2015;386(10003):1546-1555. doi:10.1016/S0140-6736(15) 61412-X

3. El-Serag HB, Mason AC. Rising incidence of hepatocellular carcinoma in the United States. $N$ Engl J Med. 1999;340(10):745-750. doi:10.1056/NEJM199903113401001

4. Forner A, Llovet JM, Bruix J. Hepatocellular carcinoma. Lancet. 2012;379(9822):1245-1255. doi:10.1016/S0140-6736(11)61347-0

5. Zhu Q, Li N, Zeng X, et al. Hepatocellular carcinoma in a large medical center of China over a 10 -year period: evolving therapeutic option and improving survival. Oncotarget. 2015;6(6):4440-4450. doi:10.18632/oncotarget.2913

6. An P, Xu J, Yu Y, Winkler CA. Host and viral genetic variation in HBV-related hepatocellular carcinoma. Front Genet. 2018;9:261. doi:10.3389/fgene.2018.00261

7. Yuen MF, Cheng CC, Lauder IJ, Lam SK, Ooi CG, Lai CL. Early detection of hepatocellular carcinoma increases the chance of treatment: Hong Kong experience. Hepatology. 2000;31(2):330-335. doi:10.1002/hep.510310211

8. Forner A, Bruix J. Biomarkers for early diagnosis of hepatocellular carcinoma. Lancet Oncol. 2012;13(8):750-751. doi:10.1016/S14702045(12)70271-1

9. Wang N, Cao Y, Song W, et al. Serum peptide pattern that differentially diagnoses hepatitis B virus-related hepatocellular carcinoma from liver cirrhosis. J Gastroenterol Hepatol. 2014;29 (7):1544-1550. doi:10.1111/jgh.12545

10. Wang $\mathrm{G}, \mathrm{Lu} \mathrm{X}, \mathrm{Du} \mathrm{Q}$, et al. Diagnostic value of the $\gamma$ glutamyltransferase and alanine transaminase ratio, alphafetoprotein, and protein induced by vitamin $\mathrm{K}$ absence or antagonist II in hepatitis B virus-related hepatocellular carcinoma. Sci Rep. 2020;10(1):13519. doi:10.1038/s41598-020-70241-5

11. Bottazzi B, Bastone A, Doni A, et al. The long pentraxin PTX3 as a link among innate immunity, inflammation, and female fertility. J Leukoc Biol. 2006;79(5):909-912. doi:10.1189/jlb.1005557

12. Bottazzi B, Doni A, Garlanda C, Mantovani A. An integrated view of humoral innate immunity: pentraxins as a paradigm. Annu Rev Immunol. 2010;28:157-183. doi:10.1146/annurev-immunol-030409101305

13. Garlanda C, Bottazzi B, Bastone A, Mantovani A. Pentraxins at the crossroads between innate immunity, inflammation, matrix deposition, and female fertility. Annu Rev Immunol. 2005;23:337-366. doi:10.1146/annurev.immunol.23.021704.115756

14. Porte R, Davoudian S, Asgari F, et al. The long pentraxin PTX3 as a humoral innate immunity functional player and biomarker of infections and sepsis. Front Immunol. 2019;10:794. doi:10.3389/ fimmu.2019.00794

15. Ristagno G, Fumagalli F, Bottazzi B, et al. Pentraxin 3 in cardiovascular disease. Front Immunol. 2019;10:823. doi:10.3389/ fimmu.2019.00823

16. Garlanda C, Bottazzi B, Magrini E, Inforzato A, Mantovani A. PTX3, a humoral pattern recognition molecule, in innate immunity, tissue repair, and cancer. Physiol Rev. 2018;98(2):623-639. doi:10.1152/ physrev.00016.2017

17. Doni A, Stravalaci M, Inforzato A, et al. The long pentraxin PTX3 as a link between innate immunity, tissue remodeling, and cancer. Front Immunol. 2019;10:712. doi:10.3389/fimmu.2019.00712

18. Song T, Wang C, Guo C, Liu Q, Zheng X. Pentraxin 3 overexpression accelerated tumor metastasis and indicated poor prognosis in hepatocellular carcinoma via driving epithelial-mesenchymal transition. $J$ Cancer. 2018;9(15):2650-2658. doi:10.7150/jca.25188

19. Carmo RF, Aroucha D, Vasconcelos LR, Pereira LM, Moura P, Cavalcanti MS. Genetic variation in PTX3 and plasma levels associated with hepatocellular carcinoma in patients with HCV. $J$ Viral Hepat. 2016;23(2):116-122. doi:10.1111/jvh.12472 
20. Deng H, Fan X, Wang X, et al. Serum pentraxin 3 as a biomarker of hepatocellular carcinoma in chronic hepatitis B virus infection. Sci Rep. 2020;10(1):20276. doi:10.1038/s41598-020-77332-3

21. El-Serag HB, Marrero JA, Rudolph L, Reddy KR. Diagnosis and treatment of hepatocellular carcinoma. Gastroenterology. 2008;134 (6):1752-1763. doi:10.1053/j.gastro.2008.02.090

22. Forner A, Reig M, Bruix J. Hepatocellular carcinoma. Lancet. 2018;391(10127):1301-1314. doi:10.1016/S0140-6736(18)30010-2

23. Mantovani A, Allavena P, Sica A, Balkwill F. Cancer-related inflammation. Nature. 2008;454(7203):436-444. doi:10.1038/ nature 07205

24. Mantovani A, Ponzetta A, Inforzato A, Jaillon S. Innate immunity, inflammation and tumour progression: double-edged swords. J Intern Med. 2019;285(5):524-532. doi:10.1111/joim.12886

25. Kumar D, Romero Y, Schuck KN, Smalley H, Subedi B, Fleming SD. Drivers and regulators of humoral innate immune responses to infection and cancer. Mol Immunol. 2020;121:99-110. doi:10.1016/j.molimm.2020.03.005

26. Martinez de la Torre Y, Fabbri M, Jaillon S, et al. Evolution of the pentraxin family: the new entry PTX4. J Immunol. 2010;184 (9):5055-5064. doi:10.4049/jimmunol.0901672

27. Giacomini A, Ghedini GC, Presta M, Ronca R. Long pentraxin 3: a novel multifaceted player in cancer. Biochim Biophys Acta Rev Cancer. 2018;1869(1):53-63. doi:10.1016/j.bbcan.2017.11.004

28. Choi B, Lee EJ, Song DH, et al. Elevated pentraxin 3 in bone metastatic breast cancer is correlated with osteolytic function. Oncotarget. 2014;5(2):481-492. doi:10.18632/oncotarget.1664

29. Diamandis EP, Goodglick L, Planque C, Thornquist MD. Pentraxin-3 is a novel biomarker of lung carcinoma. Clin Cancer Res. 2011;17 (8):2395-2399. doi:10.1158/1078-0432.CCR-10-3024
30. Choi B, Lee EJ, Park YS, et al. Pentraxin-3 silencing suppresses gastric cancer-related inflammation by inhibiting chemotactic migration of macrophages. Anticancer Res. 2015;35(5):2663-2668.

31. Kondo S, Ueno H, Hosoi $\mathrm{H}$, et al. Clinical impact of pentraxin family expression on prognosis of pancreatic carcinoma. $\mathrm{Br} J$ Cancer. 2013;109(3):739-746. doi:10.1038/bjc.2013.348

32. Stallone G, Cormio L, Netti GS, et al. Pentraxin 3: a novel biomarker for predicting progression from prostatic inflammation to prostate cancer. Cancer Res. 2014;74(16):4230-4238. doi:10.1158/00085472.CAN-14-0369

33. Zhang S, Liu Z, Wu D, Chen L, Xie L. Single-cell RNA-seq analysis reveals microenvironmental infiltration of plasma cells and hepatocytic prognostic markers in HCC with cirrhosis. Front Oncol. 2020;10:596318. doi:10.3389/fonc.2020.596318

34. Cabiati M, Gaggini M, De Simone P, et al. Do pentraxin 3 and neural pentraxin 2 have different facet function in hepatocellular carcinoma? Clin Exp Med. 2021;21(4):555-562. doi:10.1007/s10238-021-00 714-y

35. Feder S, Haberl EM, Spirk M, et al. Pentraxin-3 is not related to disease severity in cirrhosis and hepatocellular carcinoma patients. Clin Exp Med. 2020;20(2):289-297. doi:10.1007/s10238-020-00 617-4

36. Pereira JG, Silva TE, Emilia TB, et al. Circulating levels of pentraxin-3 (PTX3) in patients with liver cirrhosis. Ann Hepatol. 2017;16(5):780-787. doi:10.5604/01.3001.0010.2789

37. Luo $\mathrm{P}, \mathrm{Wu} \mathrm{S}, \mathrm{Yu} \mathrm{Y}$, et al. Current status and perspective biomarkers in AFP negative HCC: towards screening for and diagnosing hepatocellular carcinoma at an earlier stage. Pathol Oncol Res. 2020;26 (2):599-603. doi:10.1007/s12253-019-00585-5
Journal of Hepatocellular Carcinoma

\section{Publish your work in this journal}

The Journal of Hepatocellular Carcinoma is an international, peerreviewed, open access journal that offers a platform for the dissemination and study of clinical, translational and basic research findings in this rapidly developing field. Development in areas including, but not limited to, epidemiology, vaccination, hepatitis therapy, pathology and molecular tumor classification and prognostication are all considered for publication. The manuscript management system is completely online and includes a very quick and fair peer-review system, which is all easy to use. Visit http://www.dovepress.com/ testimonials.php to read real quotes from published authors. 